\title{
Compatibility of DAMA/LIBRA dark matter detection with other searches in light of new Galactic rotation velocity measurements
}

\author{
Christopher Savage, 1 * Katherine Freese, ${ }^{2, \text { 丹 }}$ Paolo Gondolo, 3 , f and Douglas Spolyar, \\ 1 William I. Fine Theoretical Physics Institute, School of Physics and Astronomy, \\ University of Minnesota, Minneapolis, MN 55455, USA \\ ${ }^{2}$ Michigan Center for Theoretical Physics, Department of Physics, \\ University of Michigan, Ann Arbor, MI 48109, USA \\ 3 Department of Physics, University of Utah, \\ 115 S 1400 E \#201 Salt Lake City, UT 84112, USA \\ 4 Department of Physics, University of California, Santa Cruz, CA, USA
}

(Dated: September 13, 2018)

\begin{abstract}
The DAMA/NaI and DAMA/LIBRA annual modulation data, which may be interpreted as a signal for the existence of weakly interacting dark matter (WIMPs) in our galactic halo, are re-examined in light of new measurements of the local velocity relative to the galactic halo. In the vicinity of the Sun, the velocity of the Galactic disk has been estimated to be $250 \mathrm{~km} / \mathrm{s}$ rather than $220 \mathrm{~km} / \mathrm{s}$ [1]. Our analysis is performed both with and without the channeling effect included. The best fit regions to the DAMA data are shown to move to slightly lower WIMP masses. Compatibility of DAMA data with null results from other experiments (CDMS, XENON10, and CRESST I) is investigated given these new velocities. A small region of spinindependent (elastic) scattering for 7-8 GeV WIMP masses remains at $3 \sigma$. Spindependent scattering off of protons is viable for $5-15 \mathrm{GeV}$ WIMP masses for direct detection experiments (but has been argued by others to be further constrained by Super-Kamiokande due to annihilation in the Sun).
\end{abstract}

PACS numbers: 95.35.+d

\section{INTRODUCTION}

Among the best motivated candidates for dark matter are Weakly Interacting Massive Particles (WIMPs). Direct searches for dark matter WIMPs seek to detect the scattering of WIMPs off of nuclei in a low-background detector. The discovery of an annual modulation by the DAMA/NaI experiment [2], confirmed by the new experiment DAMA/LIBRA [3] of the same collaboration, is the only positive signal seen in any dark matter search. The DAMA collaboration has found an annual modulation in its data compatible with the signal expected from dark matter particles bound to our Galactic Halo [4, 5]. Henceforth, we

\footnotetext{
*cmsavage@physics.umn.edu

ktfreese@umich.edu

tpaolo@physics.utah.edu

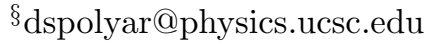


shall use the terminology "DAMA" to refer to all of the results to date of the DAMA collaboration combined. Under the assumption of elastic scattering, the DAMA data alone is compatible with two possible WIMP mass ranges: one region around $\sim 10-15 \mathrm{GeV}$ [6, 7] (due to scatters primarily off of $\mathrm{Na}$ or channeled I scatters) and another around $\sim 60-100 \mathrm{GeV}$ (due to scatters primarily off of I). The details of these allowed regions will be discussed further below. However, other direct detection experiments, e.g. CDMS [8, 9], CoGeNT [10], COUPP [11], CRESST [12, 13], TEXONO [14], and XENON10 [15, 16] have not found any signal from WIMPs. It has been difficult to reconcile a WIMP signal in DAMA with the other negative results [17], for the case of canonical WIMPs with standard weak interactions motivated by Supersymmetry (SUSY).

An alternative way to search for WIMP dark matter is via indirect detection of WIMP annihilation in the Sun or Earth [18, 19]. The most stringent indirect bounds are from SuperKamiokande [20] and will be discussed below. These bounds rely on additional physics, namely the strength of WIMP annihilation to neutrinos, and thus do not apply to all WIMPs.

Previously many authors examined this question of compatibility between all the data sets. Prior to the DAMA/LIBRA data, Ref. [6, 7] emphasized the low mass range 5-9 GeV; Ref. [21] emphasized spin-dependent interactions; Ref. [22, 23, 24] studies deviations from the standard halo assumptions; Ref. [6, 7] examined nonstandard velocity distributions (dark matter streams); Ref. 25] proposed inelastic WIMP/nucleon scattering. In the past year DAMA/LIBRA reported new experimental results, including presenting the modulation data in 36 separate energy bins and taking into account the possibility of ion channeling.

Many authors studied the new DAMA data together with the null results of other experiments, and included the effects of ion channeling: [24, 26, 27, 28, 29, 30, 31, 32] (although other non-WIMP candidates were studied as well, such as mirror [33], composite [34], and WIMPless [35] dark matter. Non-neutralino WIMPs and Higgs exchange are examined in Ref. [32]). In our previous work [26], we delineated the remaining regions of parameter space using the velocity information about our motion relative to the Galactic Halo available at that time.

Recent work reexamined the local disk rotational velocities. They examined star-forming regions [36, 37, 38, 39] and found that the fit to their data is significantly improved if the local velocity is taken to be higher than previously thought [1]. This shift in the velocity has the potential to change the interpretation of the DAMA modulation signal as well as signals from other experiments. It is the purpose of this paper to examine this effect. We restrict our studies to the standard isothermal model for the dark matter halo. We investigate both spin-independent and spin-dependent coupling of WIMP/nucleus elastic scattering, with and without channeling included. First, we find the new "best fit" regions for the DAMA data. Then, we compare with null results from other experiments via the likelihood method with goodness-of-fit.

We begin by reviewing dark matter detection, including the role of the new velocity information, in Section II. In Section III we review the data from DAMA and other experiments. We do not present much detail here, as we are using the same data and analysis techniques that we discussed in our previous paper [26]. In Section [V] we apply our analysis techniques to obtain our results, which can primarily be found in our figures. 


\section{DARK MATTER DETECTION}

WIMP direct detection experiments seek to measure the energy deposited when a WIMP interacts with a nucleus in the detector [40]. If a WIMP of mass $m$ scatters elastically from a nucleus of mass $M$, it will deposit a recoil energy $E=\left(\mu^{2} v^{2} / M\right)(1-\cos \theta)$, where $\mu \equiv m M /(m+M)$ is the reduced mass, $v$ is the speed of the WIMP relative to the nucleus, and $\theta$ is the scattering angle in the center of mass frame. The differential recoil rate per unit detector mass for a WIMP mass $m$, typically given in units of $\mathrm{cpd} / \mathrm{kg} / \mathrm{keV}$ (where cpd is counts per day), can be written as:

$$
\frac{\mathrm{d} R}{\mathrm{~d} E}=\frac{\sigma(q)}{2 m \mu^{2}} \rho \eta(E, t)
$$

where $q=\sqrt{2 M E}$ is the nucleus recoil momentum, $\sigma(q)$ is the WIMP-nucleus cross-section, $\rho$ is the local WIMP density, and information about the WIMP velocity distribution is encoded into the mean inverse speed $\eta(E, t)$,

$$
\eta(E, t)=\int_{u>v_{\min }} \frac{f(\mathbf{u}, t)}{u} \mathrm{~d}^{3} u .
$$

Here

$$
v_{\min }=\sqrt{\frac{M E}{2 \mu^{2}}}
$$

represents the minimum WIMP velocity that can result in a recoil energy $E$ and $f(\mathbf{u}, t)$ is the (time-dependent) distribution of WIMP velocities $\mathbf{u}$ relative to the detector.

To determine the number of expected recoils for a given experiment and WIMP mass, we integrate Eqn. (11) over the nucleus recoil energy to find the recoil rate $R$ per unit detector mass:

$$
R(t)=\int_{E_{1} / Q}^{E_{2} / Q} \mathrm{~d} E \epsilon(Q E) \frac{\rho}{2 m \mu^{2}} \sigma(q) \eta(E, t) .
$$

$\epsilon(Q E)$ is the (energy dependent) efficiency of the experiment, due, e.g., to data cuts designed to reduce backgrounds. $Q$ is the quenching factor relating the observed energy $E_{\text {det }}$ (in some cases referred to as the electron-equivalent energy) with the actual recoil energy $E_{\text {rec }}$ : $E_{\text {det }}=Q E_{\text {rec }}$ (explained in more detail below). The energy range between $E_{1}$ and $E_{2}$ is that of observed energies for some data bin of the detector (where experiments often bin observed recoils by energy). The quenching factor $Q$ depends on the nuclear target and the characteristics of the detector.

For detectors with multiple elements and/or isotopes, the total rate is given by:

$$
R_{\text {tot }}(t)=\sum_{i} f_{i} R_{i}(t)
$$

where $f_{i}$ is the mass fraction and $R_{i}$ is the rate (Eqn. (4I)) for element/isotope $i$.

The expected number of recoils observed by a detector is given by:

$$
N_{r e c}=M_{d e t} T R
$$

where $M_{d e t}$ is the detector mass and $T$ is the exposure time. 


\section{A. Cross-Section}

The $\sigma(q)$ cross-section term in Eqns. (11) \& (41) is an effective cross-section for scatters with a momentum exchange $q$. The momentum exchange dependence appears in form factors that arise from the finite size of the nucleus. The total scattering cross-section generally has contributions from spin-independent (SI) and spin-dependent (SD) couplings, with

$$
\sigma=\sigma_{\mathrm{SI}}+\sigma_{\mathrm{SD}}
$$

these two cross-sections are described below.

Spin-independent (SI). For spin-independent WIMP interactions, we make the usual assumption [41] that the cross section $\sigma$ scales with the square of the nucleus atomic number $A$ and is given by

$$
\sigma=\sigma_{0}|F(E)|^{2}
$$

where $\sigma_{0}$ is the zero-momentum WIMP-nuclear cross-section and $F(E)$ is a nuclear form factor, normalized to $F(0)=1$. For purely scalar interactions,

$$
\sigma_{0, \mathrm{SI}}=\frac{4 \mu^{2}}{\pi}\left[Z f_{\mathrm{p}}+(A-Z) f_{\mathrm{n}}\right]^{2} .
$$

Here $Z$ is the number of protons, $A-Z$ is the number of neutrons, and $f_{\mathrm{p}}$ and $f_{\mathrm{n}}$ are the WIMP couplings to the proton and nucleon, respectively. In most instances, $f_{\mathrm{n}} \sim f_{\mathrm{p}}$; the WIMP-nucleus cross-section can then be given in terms of the WIMP-proton cross-section as a result of Eqn. (9):

$$
\sigma_{0, \mathrm{SI}}=\sigma_{\mathrm{p}, \mathrm{SI}}\left(\frac{\mu}{\mu_{\mathrm{p}}}\right)^{2} A^{2}
$$

where the $\mu_{\mathrm{p}}$ is the proton-WIMP reduced mass, and $A$ is the atomic mass of the target nucleus. In this model, for a given WIMP mass, $\sigma_{\mathrm{p}, \mathrm{SI}}$ is the only free parameter. For the nuclear form factor we use the conventional Helmi form [41, 42, 43].

Spin-dependent (SD). The generic form for the spin-dependent WIMP-nucleus crosssection includes two couplings [44], the WIMP-proton coupling $a_{p}$ and the WIMP-neutron coupling $a_{n}$,

$$
\sigma_{S D}(q)=\frac{32 \mu^{2} G_{F}^{2}}{2 J+1}\left[a_{p}^{2} S_{p p}(q)+a_{p} a_{n} S_{p n}(q)+a_{n}^{2} S_{n n}(q)\right]
$$

Here, the quantities $a_{p}$ and $a_{n}$ are actually the axial four-fermion WIMP-nucleon couplings in units of $2 \sqrt{2} G_{F}$ [45, 46, 47]. The nuclear structure functions $S_{p p}(q), S_{n n}(q)$, and $S_{p n}(q)$ are functions of the exchange momentum $q$ and are specific to each nucleus. We take the structure functions for Aluminum from Ref. [48]; for Sodium, Iodine, and Xenon from Ref. [49]; and for Silicon and Germanium from Ref. [50]. These and additional structure functions may be found in the review of Ref. [51].

\section{B. Velocity Distribution}

We assume the Standard Halo Model (SHM) [5], in which the Galactic Halo is a simple non-rotating isothermal sphere. We use a Maxwellian distribution, characterized by an rms 
velocity dispersion $\sigma_{v}$, to describe the WIMP speeds, and we will allow for the distribution to be truncated at some escape velocity $v_{\text {esc }}$,

$$
\widetilde{f}(\mathbf{v})= \begin{cases}\frac{1}{N_{\mathrm{esc}}}\left(\frac{3}{2 \pi \sigma_{v}^{2}}\right)^{3 / 2} e^{-3 \mathbf{v}^{2} / 2 \sigma_{v}^{2}}, & \text { for }|\mathbf{v}|<v_{\mathrm{esc}} \\ 0, & \text { otherwise. }\end{cases}
$$

Here

$$
N_{\text {esc }}=\operatorname{erf}(z)-2 z \exp \left(-z^{2}\right) / \pi^{1 / 2},
$$

with $z \equiv v_{\text {esc }} / \bar{v}_{0}$, is a normalization factor. The most probable speed,

$$
\bar{v}_{0}=\sqrt{2 / 3} \sigma_{v},
$$

is used to generate unitless parameters such as $z$. For distributions without an escape velocity $\left(v_{\text {esc }} \rightarrow \infty\right), N_{\text {esc }}=1$.

The WIMP component (halo or stream) often exhibits a bulk motion relative to us, so that

$$
f(\mathbf{u})=\widetilde{f}\left(\mathbf{v}_{\text {obs }}+\mathbf{u}\right),
$$

where $\mathbf{v}_{\text {obs }}$ is the motion of the observer relative to the rest frame of the WIMP component described by Eqn. (12); this motion will be discussed below. For the truncated Maxwellian velocity distribution in Eqn. (12), the mean inverse speed, Eqn. (21), becomes

$$
\eta(E, t)= \begin{cases}\frac{1}{\bar{v}_{0} y}, & \text { for } z<y, x<|y-z| \\ \frac{1}{2 N_{\text {esc }} \bar{v}_{0} y}\left[\operatorname{erf}(x+y)-\operatorname{erf}(x-y)-\frac{4}{\sqrt{\pi}} y e^{-z^{2}}\right], & \text { for } z>y, x<|y-z| \\ \frac{1}{2 N_{\text {esc }} \bar{v}_{0} y}\left[\operatorname{erf}(z)-\operatorname{erf}(x-y)-\frac{2}{\sqrt{\pi}}(y+z-x) e^{-z^{2}}\right], & \text { for }|y-z|<x<y+z \\ 0, & \text { for } y+z<x\end{cases}
$$

where

$$
x \equiv v_{\min } / \bar{v}_{0}, \quad y \equiv v_{\mathrm{obs}} / \bar{v}_{0}, \quad \text { and } \quad z \equiv v_{\mathrm{esc}} / \bar{v}_{0} ;
$$

recall $v_{\min }$ is given by Eqn. (3). Here, we use the common notational convention of representing 3-vectors in bold and the magnitude of a vector in the non-bold equivalent, e.g. $v_{\text {obs }} \equiv\left|\mathbf{v}_{\text {obs }}\right|$.

Due to the motion of the Earth around the Sun, $\mathbf{v}_{\text {obs }}$ is time dependent: $\mathbf{v}_{\text {obs }}=\mathbf{v}_{\text {obs }}(t)$. We write this in terms of the Earth's velocity $\mathbf{V}_{\oplus}$ relative to the Sun as

$$
\mathbf{v}_{\text {obs }}(t)=\mathbf{v}_{\odot}+V_{\oplus}\left[\hat{\boldsymbol{\varepsilon}}_{1} \cos \omega\left(t-t_{1}\right)+\hat{\boldsymbol{\varepsilon}}_{2} \sin \omega\left(t-t_{1}\right)\right],
$$

where $\omega=2 \pi /$ year, $\mathbf{v}_{\odot}$ is the Sun's motion relative to the WIMP component's rest frame, $V_{\oplus}=29.8 \mathrm{~km} / \mathrm{s}$ is the Earth's orbital speed, and $\hat{\varepsilon}_{1}$ and $\hat{\varepsilon}_{2}$ are the directions of the Earth's velocity at times $t_{1}$ and $t_{1}+0.25$ years, respectively. With this form, we have neglected the ellipticity of the Earth's orbit, although the ellipticity is small and, if accounted for, would give only negligible changes in the results of this paper (see Refs. [23, 42] for more detailed expressions). For clarity, we have used explicit velocity vectors rather than the position vectors $\hat{\mathbf{e}}_{1}$ and $\hat{\mathbf{e}}_{2}$ used in Refs. [52, 53] and elsewhere (the position vectors are more easily generalized to an elliptical orbit); the two bases are related by $\hat{\boldsymbol{\varepsilon}}_{1}=-\hat{\mathbf{e}}_{2}$ and $\hat{\boldsymbol{\varepsilon}}_{2}=\hat{\mathbf{e}}_{1}$. 
In Galactic coordinates, where $\hat{\mathbf{x}}$ is the direction to the Galactic Center, $\hat{\mathbf{y}}$ the direction of disk rotation, and $\hat{\mathbf{z}}$ the North Galactic Pole,

$$
\begin{aligned}
& \hat{\varepsilon}_{1}=(0.9931,0.1170,-0.01032), \\
& \hat{\varepsilon}_{2}=(-0.0670,0.4927,-0.8676),
\end{aligned}
$$

where we have taken $\hat{\varepsilon}_{1}$ and $\hat{\varepsilon}_{2}$ to be the direction of the Earth's motion at the Spring equinox (March 21, or $t_{1}$ ) and Summer solstice (June 21), respectively.

\section{New Measurement: Local Standard of Rest}

Unlike the Galactic disk (and the Sun), the halo has essentially no rotation; the motion of the Sun relative to this stationary halo is

$$
\mathbf{v}_{\odot, \mathrm{SHM}}=\mathbf{v}_{\mathrm{LSR}}+\mathbf{v}_{\odot, \mathrm{pec}}
$$

where $\mathbf{v}_{\mathrm{LSR}}$ is the motion of the Local Standard of Rest and $\mathbf{v}_{\odot, \text { pec }}=(10,13,7) \mathrm{km} / \mathrm{s}$ is the Sun's peculiar velocity (i.e. the so-called Solar Motion). Previously, the value for the Local Standard of Rest was taken to be $\mathbf{v}_{\mathrm{LSR}}=(0,220,0) \mathrm{km} / \mathrm{s}$. Now, however, new measurements have been made [36, 37, 38, 39] and the speed that provides the best fit to data has been found to be higher: $\mathbf{v}_{\mathrm{LSR}}=(0,250,0) \mathrm{km} / \mathrm{s}$. This change in velocity leads to the effects examined in this paper. For an isothermal halo, the most probable speed in Eqn. (14) is the same as the circular velocity, i.e., $\bar{v}_{0}=250 \mathrm{~km} / \mathrm{s}$; thus the velocity dispersion is taken to be $\sigma_{v}=306 \mathrm{~km} / \mathrm{s}$. The best fit for the Sun's peculiar velocity may also shift slightly, but the changes due to this effect are much smaller and we will ignore them. Though the escape velocity from the Milky Way may also change (and is the subject of a future work), the uncertainties are so great that we continue to use the standard value $v_{\text {esc }}=650 \mathrm{~km} / \mathrm{s}$. We truncate the distribution in Eqn. (12) at this value.

\section{Annual Modulation}

The count rate in WIMP detectors will experience an annual modulation as a result of the motion of the Earth around the Sun described above [4, 5]. Typically the count rate (Eqn. (1)) has an approximate time dependence

$$
\frac{\mathrm{d} R}{\mathrm{~d} E}(E, t) \approx S_{0}(E)+S_{m}(E) \cos \omega\left(t-t_{c}\right)
$$

where $t_{c}$ is the time of year at which $v_{\text {obs }}(t)$ is at its maximum. $S_{0}(E)$ is the average differential recoil rate over a year and $S_{m}(E)$ is referred to as the modulation amplitude (which may, in fact, be negative). The above equation is a reasonable approximation for the SHM we are considering in this paper, but is not valid for all halo models, particularly at some recoil energies for dark matter streams; see Ref. [54] for a discussion. For the SHM,

$$
S_{m}(E)=\frac{1}{2}\left[\frac{\mathrm{d} R}{\mathrm{~d} E}(E, \text { June } 1)-\frac{\mathrm{d} R}{\mathrm{~d} E}(E, \text { Dec } 1)\right]
$$


Experiments such as DAMA will often give the average amplitude over some interval,

$$
S_{m}=\frac{1}{E_{2}-E_{1}} \int_{E_{1}}^{E_{2}} \mathrm{~d} E S_{m}(E) .
$$

The Earth's speed relative to the halo, $v_{\text {obs }}(t)$, is maximized around June 1 . While this date varies with changes in the velocity distribution, the change is negligible (less than a day) for the values we are considering.

\section{E. Parameter Space}

Many of the parameters that factor into the expected recoil rates for a scattering detector are unknown, including the WIMP mass, four WIMP-nucleon couplings (SI and SD couplings to each of protons and neutrons), the local WIMP density, and the WIMP velocity distribution in the halo. In this paper, we shall fix the halo model to the SHM and take the local dark matter density $\rho_{0}$ to be the estimated average density in the local neighborhood, $0.3 \mathrm{GeV} / \mathrm{cm}^{3}$. In addition, we shall take $f_{\mathrm{p}}=f_{\mathrm{n}}$ (equal SI couplings) so that there are only three independent scattering couplings; the SI coupling will be given in terms of the SI scattering cross-section off the proton, $\sigma_{\mathrm{p}, \mathrm{SI}}$. The parameter space we examine will then consist of the four parameters $m, \sigma_{\mathrm{p}, \mathrm{SI}}, a_{\mathrm{p}}$, and $a_{\mathrm{n}}$.

\section{EXPERIMENTS}

\section{A. DAMA}

The DAMA experiments remain the only direct detection experiments to observe a signal. We primarily focus on the annual modulation signal found by the DAMA group. Details of our analysis can be found in our previous paper [26].

Ion Channeling $(I C)$ : Here we review the experimental effect of ion channeling, which has been found to affect the results. In general only a fraction (known as the quenching factor) of the recoil energy deposited by a WIMP is transferred to electrons and is converted into useful signal in the DAMA detector (e.g. ionization or scintillation); the remainder is converted into phonons and heat and goes undetected. Hence measured energies must be corrected for this behavior to obtain the proper recoil energy, by dividing by this quenching factor. The DAMA detector is composed of NaI, with quenching factors $Q_{N a}=0.3$ and $Q_{I}=0.09$. Yet, recently, the DAMA collaboration pointed out that some fraction of the nuclei may recoil along a crystal axis or plane [55], in which cases the recoiling nucleus can travel relatively large distances along the "channels" between the crystal atoms (as opposed to the typical case, where the recoiling nucleus quickly collides with nearby atoms). Such recoiling nuclei lose very little energy to other atoms and to heat, giving nearly all their energy to electrons. For these cases the measured energy corresponds very nearly to the energy deposited by the WIMP and thus have quenching factor $Q \approx 1$. As a consequence, the detector is sensitive to lower mass WIMPs than previously thought. The new channeling studies revived the possiblity of DAMA's compatibility with other data sets, particularly at low masses. We shall examine the DAMA results both with and without including the IC effect. Details of our analysis can be found in our previous paper [26]. 
Total Rate: While we mainly examine the DAMA experiment by analyzing the modulation amplitude $S_{m}$, DAMA can additionally constrain parameter space using the total rate $\left(S_{0}\right.$ in Eqn. (22) ) as shown in Figure 1 of Ref. [3]. The DAMA detectors do not strongly discriminate between WIMP scatters and background events, so an unknown and possibly large portion of the total observed events may be due to backgrounds (the backgrounds are presumed not to vary with time and do not contribute to the modulation). In the same manner as the various null experiments, we use the total number of events observed by DAMA to constrain the parameter space by excluding regions that would predict more events than observed.

We will show regions excluded at the $90 \%$ level due to the total rate for DAMA. Constraints will be shown with and without the channeling effect. Note that some choices of parameters that produce the correct modulation signal may actually predict a total rate larger than observed and, hence, those parameter choices are incompatible with the full DAMA data set.

\section{B. Null Results}

Numerous other experiments have searched for a dark matter signal, but all these experimental results, apart from DAMA, are consistent with no WIMP signal.

Direct Detection: In our previous paper [26] we discussed in detail our use of data from many experiments. We do not repeat this information here. In this paper we restrict our analysis to the most constraining experiments: CDMS [9] [8], XENON [15, 16], and CRESST I [12]. While COUPP [11] could be very powerful, insufficient information on the data runs is provided to reproduce their results and extend them to arbitrary couplings.

For the various null experiments, we define constraints in parameter space at a certain exclusion level $1-\alpha$, typically $90 \%$, as the parameters for which the probability of seeing the experimental result is $\alpha$. Parameters outside the corresponding contours would yield the observed result with a probability less than $\alpha$. We say the parameters within those contours are compatible within the $1-\alpha$ level and parameters outside are excluded at the $1-\alpha$ level. The probabilities are determined using different statistical values, described in our previous paper, in different cases.

Indirect Detection: An alternative way to search for WIMP dark matter is via indirect detection of WIMP annihilation in the Sun or Earth. When WIMPs pass through these objects [18, 19], a small fraction may be captured, sink to the core, and annihilate with one another to produce a neutrino signal observable in Earth-based detectors such as SuperKamiokande [20] (Super-K), AMANDA [56, 57], IceCube [58] and ANTARES [59] ${ }^{1}$. Since the Sun is mostly made of hydrogen (which has spin), its capture of WIMPs depends primarily on the spin-dependent WIMP/nucleon cross section which can thus be constrained. The most stringent indirect bounds are from Super-K [20, 31]. However, Super-K is not shown in the plots because their result cannot be readily reanalyzed for a modified halo.

The Super-K constraints rely on the following assumptions: (1) the WIMP/anti-WIMP abundance is not highly asymmetric, which would suppress the annihilation rate; (2) the

1 Other indirect detection methods search for WIMPs that annihilate in the Galactic Halo or near the Galactic Center where they produce neutrinos, positrons, or antiprotons that may be seen in detectors on the Earth [60, 61]. 
annihilation cross-section is sufficiently high so that the capture rate of WIMPs in the Sun (via scattering off of nuclei in the Sun) is in equilibrium with the annihilation rate; and (3) the WIMP does not annihilate predominantly into the light quarks, which do not yield neutrinos in sufficient quantities and energies to be observed. For the Constrained Minimal Supersymmetric Standard Model, these assumptions are mainly satisfied for neutralinos in the parameter space of interest. In general, however, these assumptions need not be satisfied, so the Super-K constraints should be applied with caution.

\section{Analysis Techniques}

We use two statistical tests to interpret the DAMA modulation data. First, we find the "best fit" regions in WIMP parameter space to fit the DAMA data by itself. More specifically, we use a likelihood ratio method with a global fit of four parameters to find the preferred parameters to produce the DAMA signal, and plot these in the plane of the WIMP/nucleon scattering cross section vs. WIMP mass. Second, we use a $\chi^{2}$ goodness-offit test (g.o.f.) to indicate which parameters are compatible with the DAMA signal. See Ref. [62] for a short review of statistics or Ref. [63] for more extensive discussions.

\section{Best Fit to DAMA data (Likelihood Ratio Method)}

To determine the most likely parameters for producing the DAMA signal, we use the maximum likelihood method, based on the likelihood ratio

$$
\frac{L\left(S_{m, k} \mid m, \sigma_{\mathrm{p}, \mathrm{SI}}, a_{\mathrm{p}}, a_{\mathrm{n}}\right)}{L\left(S_{m, k} \mid \hat{m}, \hat{\sigma}_{\mathrm{p}, \mathrm{SI}}, \hat{a}_{\mathrm{p}}, \hat{a}_{\mathrm{n}}\right)},
$$

where $L$ is the likelihood function, $S_{m, k}$ are the observed modulation amplitudes in each bin, and $\hat{m}, \hat{\sigma}_{\mathrm{p}, \mathrm{SI}}, \hat{a}_{\mathrm{p}}$, and $\hat{a}_{\mathrm{n}}$ are the values of the parameters that maximize the likelihood for the observed $S_{m, k}$. The denominator of the above equation is the maximum likelihood value $L_{\max }$. Confidence regions in the parameters are determined by

$$
2 \ln L\left(m, \sigma_{\mathrm{p}, \mathrm{SI}}, a_{\mathrm{p}}, a_{\mathrm{n}}\right) \geq 2 \ln L_{\max }-2 \Delta \ln L,
$$

where the value of $\Delta \ln L$ corresponds to the confidence level (C.L.) of the confidence region. Since each bin is normally distributed, this equation may instead by given in terms of the $\chi^{2}$,

$$
\chi^{2}\left(m, \sigma_{\mathrm{p}, \mathrm{SI}}, a_{\mathrm{p}}, a_{\mathrm{n}}\right) \leq \chi_{\min }^{2}+\Delta \chi^{2},
$$

where

$$
\chi^{2}\left(m, \sigma_{\mathrm{p}, \mathrm{SI}}, a_{\mathrm{p}}, a_{\mathrm{n}}\right) \equiv \sum_{k} \frac{\left(S_{m, k}-S_{m, k}^{\mathrm{Th}}\right)^{2}}{\sigma_{k}^{2}},
$$

$\sigma_{k}$ is the uncertainty in $S_{m, k}, S_{m, k}^{\mathrm{Th}} \equiv S_{m, k}^{\mathrm{Th}}\left(m, \sigma_{\mathrm{p}, \mathrm{SI}}, a_{\mathrm{p}}, a_{\mathrm{n}}\right)$ is the expected amplitude in a particular bin for the given parameters, and $\chi_{\min }^{2}$ is the minimum value of $\chi^{2}$.

The $\Delta \chi^{2}$ limit corresponding to different C.L.'s depends upon the number of parameters that have been minimized and, in the large data sample limit, is determined from a $\chi^{2}$ distribution with the degrees of freedom (d.o.f.) equal to the number of minimized parameters. 
For the four parameters considered here, $\Delta \chi^{2}=7.8$ (90\% C.L.), $16.3(3 \sigma), 34.6(5 \sigma)$, and $60.3(7 \sigma)$.

The confidence region for DAMA as described here is, in fact, a 4-dimensional region in the $\left(m, \sigma_{\mathrm{p}, \mathrm{SI}}, a_{\mathrm{p}}, a_{\mathrm{n}}\right)$ parameter space. We shall be showing 2-dimensional slices of this larger region for particular cases, such as the $a_{\mathrm{p}}=a_{\mathrm{n}}=0$ slice corresponding to SI only scattering. This is not equivalent to fixing $a_{\mathrm{p}}=a_{\mathrm{n}}=0$ and determining a 2-dimensional confidence region by minimizing only over $\left(m, \sigma_{\mathrm{p}, \mathrm{SI}}\right)$. In the latter case, a confidence region will always be defined in a 2-dimensional plane while, in the former, there may be no slice in that plane if that plane represents a poor "fit" relative to the overall parameter space.

Note, as will be discussed in Section IV, the confidence regions obtained via this method yield the most preferred parameters for producing the signal. This method, in and of itself, does not imply parameters outside the confidence regions are necessarily a bad fit to the data (or, conversely, that parameters inside these regions are a good fit) and one should be careful using these regions to compare with other experiments ${ }^{2}$.

\section{Goodness-of-Fit}

We use the alternative statistic of goodness-of-fit (g.o.f.) to indicate when parameters are incompatible with DAMA data, as opposed to finding the best fit region as we did previously. This statistic allows for comparison of DAMA modulation data both with the total count rate from the DAMA data itself and with null results from other experiments (whereas it would be inappropriate to use the best fit regions for this purpose). To conservatively indicate parameters that are statistically compatible with the DAMA data set, this method indicates regions at which the $\chi^{2}$ falls within a given level using a simple $\chi^{2}$ goodness-of-fit (g.o.f.) test on the data. In contrast to the previous analysis method, there is no fit to the parameters here. The g.o.f. regions are defined as those parameters for which

$$
\chi^{2}\left(m, \sigma_{\mathrm{p}, \mathrm{SI}}, a_{\mathrm{p}}, a_{\mathrm{n}}\right) \leq \chi_{\mathrm{GOF}}^{2},
$$

where $\chi_{\mathrm{GOF}}^{2}$ is the value at which the $\chi^{2}$ cumulative distribution function (CDF) for e.g. 36 d.o.f. is equal to the desired level of compatibility. That is, for a desired compatibility level of $1-\alpha$, there is a probability $\alpha$ that $\chi^{2}$ will exceed $\chi_{\text {GOF }}^{2}$. Alternatively, we can say parameters outside of the region are excluded at the $1-\alpha$ level.

To improve the ability of the g.o.f. test to exclude some parameters, we combine all DAMA bins over 10-20 keVee into a single bin (energies at which negligible signal is expected), resulting in a total of 17 data bins used for this test. For the 17 d.o.f. $\chi^{2}$ distribution, values of $24.8,37.7$, and 61.6 are excluded at the $90 \%, 3 \sigma$, and $5 \sigma$ levels, respectively.

While the g.o.f. regions defined here are, in fact, confidence regions - indicating the likely parameters to produce the DAMA signal in our theoretical framework - we do not use these regions in that manner. The determination of a confidence region assumes that the theoretical framework is correct, i.e. there exists some choice of parameters that is correct. That assumption may not be valid if, e.g., the standard halo model is not a reasonable approximation of the actual halo. Instead, we will more conservatively only use this g.o.f. test to exclude parameters outside of the corresponding regions. That is, parameters outside of the

\footnotetext{
${ }^{2}$ In statistical parlance, the determination of the confidence region for this method is decoupled from the goodness-of-fit.
} 

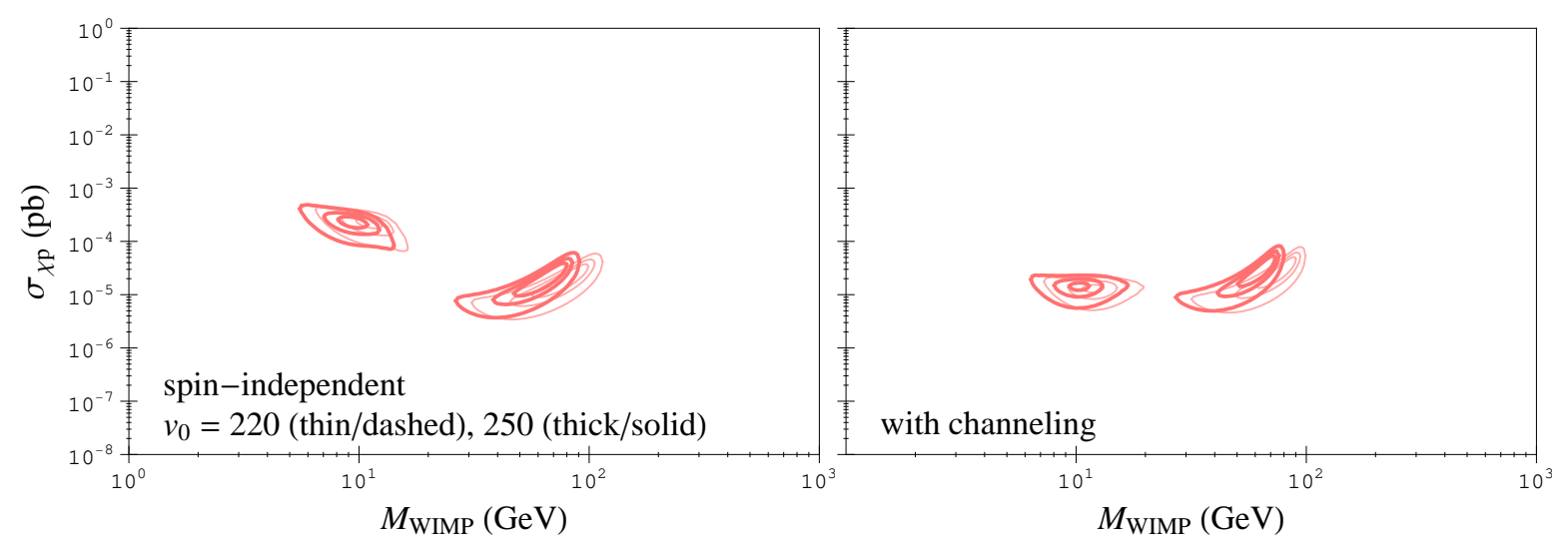

FIG. 1: DAMA best fit parameters (cross section and WIMP mass) for SI only scattering. The DAMA contours are shown at $5 \sigma, 3 \sigma$, and $90 \%$ C.L. (outermost contour is $5 \sigma$ ). Light/thin lines are for the old value of local standard of rest $\left(v_{0}=220 \mathrm{~km} / \mathrm{s}\right)$ while the dark/thick lines are the new value $\left(v_{0}=250 \mathrm{~km} / \mathrm{s}\right)$. The DAMA preferred regions are determined without (Panel A) and with (Panel B) the channeling effect.

DAMA g.o.f. regions are incompatible with the DAMA signal at the given level within the context of this model.

\section{RESULTS}

The likelihood ratio analysis of DAMA yields a 4-dimensional confidence region over the $\left(m, \sigma_{\mathrm{p}, \mathrm{SI}}, a_{\mathrm{p}}, a_{\mathrm{n}}\right)$ parameter space. In Figures 1, 3, and 5, we show the SI only $\left(a_{\mathrm{p}}=a_{\mathrm{n}}=0\right)$, $\mathrm{SD}$ proton-only $\left(\sigma_{\mathrm{p}, \mathrm{SI}}=a_{\mathrm{n}}=0\right)$, and $\mathrm{SD}$ neutron-only $\left(\sigma_{\mathrm{p}, \mathrm{SI}}=a_{\mathrm{p}}=0\right)$ slices of this confidence region, respectively. The thin/dashed curves are for the old velocity of the local standard of rest $v_{0}=220 \mathrm{~km} / \mathrm{s}$ while the solid curves are for the new local standard of rest $v_{0}=250 \mathrm{~km} / \mathrm{s}$. In all cases, the first panel of any figure is without channeling, while the second panel is with channeling taken into account for DAMA.

We also present results using the goodness-of-fit statistic to compare DAMA's positive annual modulation signal with its own bound on the total count rate plus null results from other experiments (CRESST I, CDMS, and XENON10) to find what regions of parameter space remain compatible with all of these direct detection searches. The total rate from DAMA itself restricts the high mass end, and in some cases rules out the entire higher mass window (near $60 \mathrm{GeV}$ ).

While we present our results in three particular cases - SI only, SD proton-only, and SD neutron-only - it is important to keep in mind that there is a larger region of parameter space that includes mixed couplings.

\section{A. Spin-Independent Couplings (SI)}

Figure 1 shows the best fit regions to the DAMA data for spin-independent couplings, both with and without channeling. One can see that the effect of the larger velocity is to 

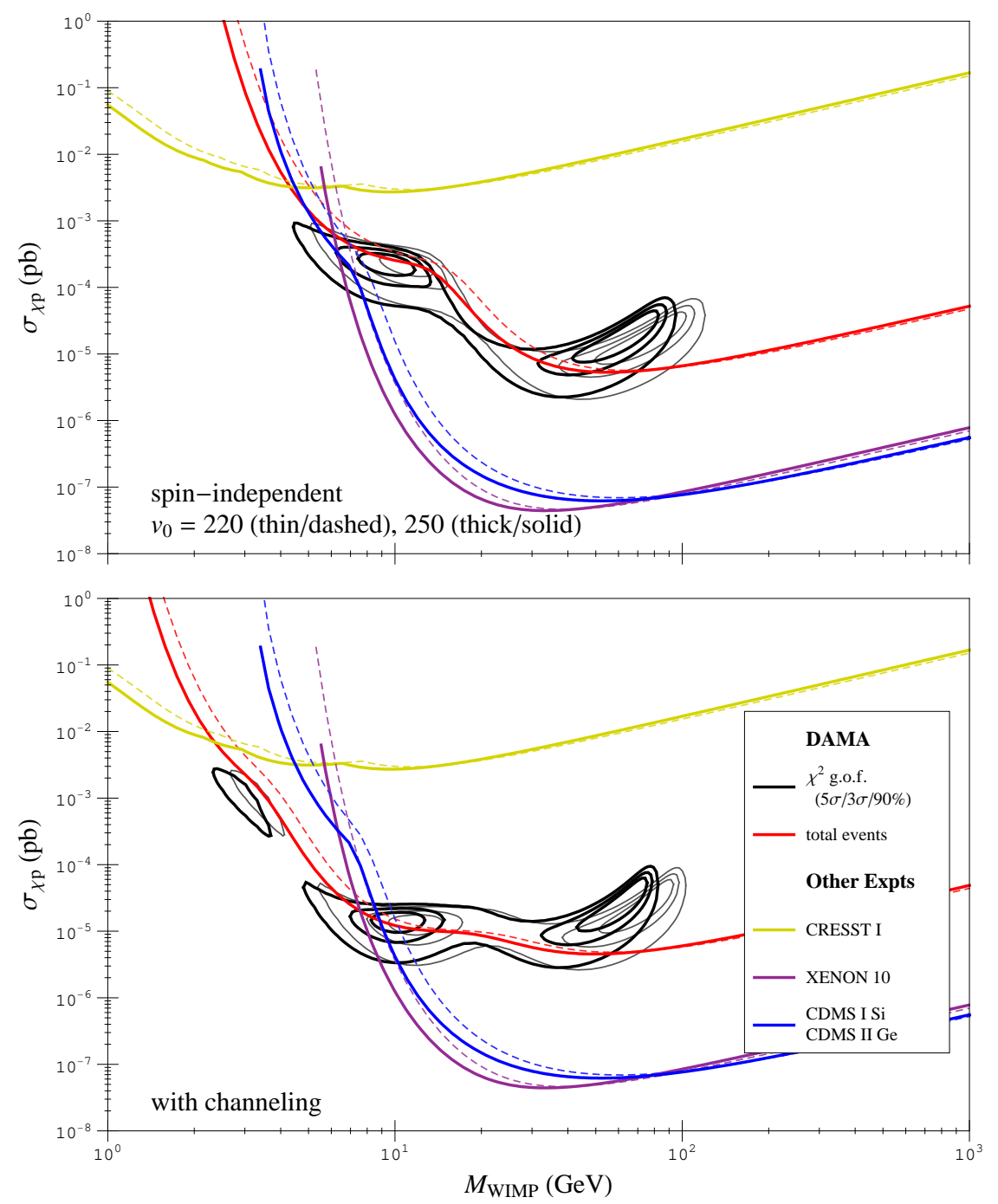

FIG. 2: Spin-independent WIMP/nucleon scattering cross section vs. WIMP mass: comparison of DAMA with other null experiments (CRESST I, XENON10, and CDMS) using the goodness-of-fit statistic. Thin/dashed lines are for the old value of local standard of rest $\left(v_{0}=220 \mathrm{~km} / \mathrm{s}\right)$ while the thick/solid lines are the new value $\left(v_{0}=250 \mathrm{~km} / \mathrm{s}\right)$. The bound from the total events from DAMA is also shown (red solid line). Panel A does not include the effects of ion channeling while Panel B does take it into account. The DAMA contours are shown at $5 \sigma, 3 \sigma$, and 90\% C.L. (outermost contour is $5 \sigma)$. One can see that, with IC included, a sliver of parameter space remains viable at $3 \sigma$ for $7-8 \mathrm{GeV}$ WIMPs.

shift the regions to slightly lower masses. Figure 2 uses the goodness-of-fit (g.o.f.) statistic to compare the DAMA regions with the null experiments, for the SI couplings. Most of the parameter space is incompatible, with the exception of a small sliver at 7-8 GeV WIMP masses that are still in agreement with all experiments to $3 \sigma$. 

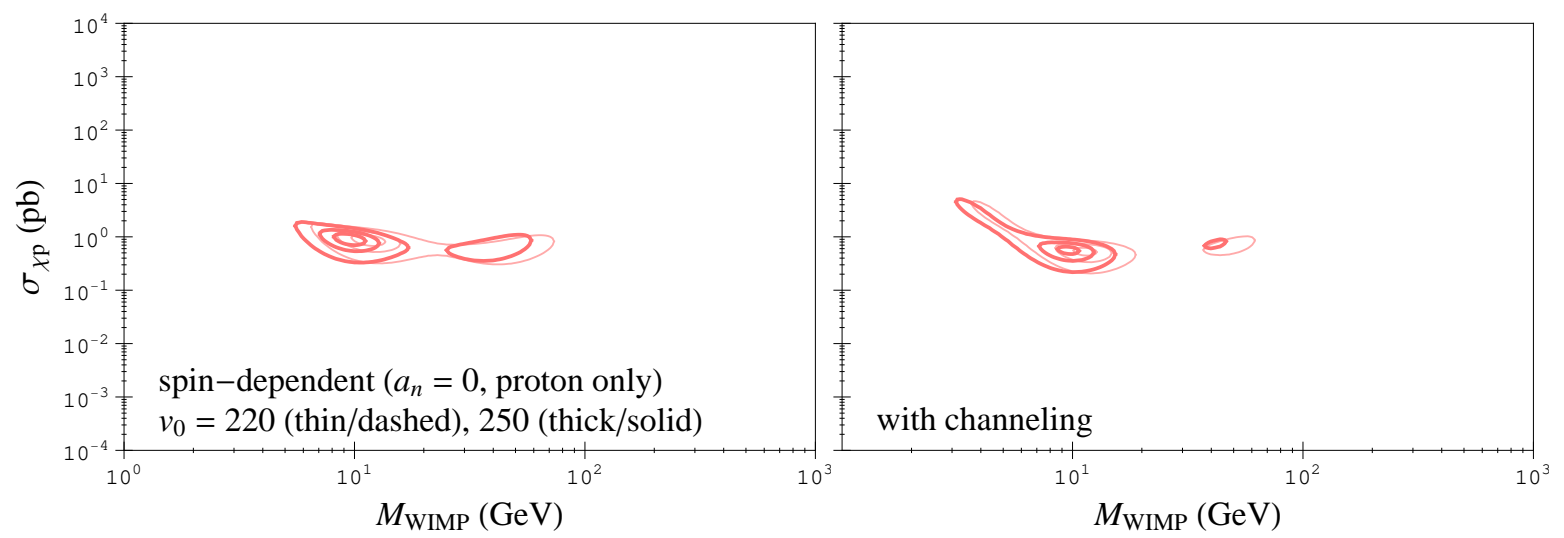

FIG. 3: Same as Figure 1 but for SD proton-only scattering $\left(a_{n}=0\right)$. Note there are no $3 \sigma$ or $90 \%$ C.L. contours at the higher WIMP mass region.

\section{B. Spin-Dependent Couplings (SD)}

Proton only $\left(a_{n}=0\right)$ : Figure 3 shows the best fit regions to the DAMA data for spindependent couplings for proton only $\left(a_{n}=0\right)$, both with and without channeling. Again, the best fit regions move to slightly lower masses. In Figure 4 one can see that all WIMP masses suitable for the DAMA data are compatible with all the other direct detection experiments. With channeling, the $90 \%$ region is for $7-12 \mathrm{GeV}$ WIMP masses and the $3 \sigma$ region is for 5-15 GeV WIMP masses. However, the COUPP results, once made available, can severely change these conclusions.

The Super-K indirect detection results, which have not been plotted, can also change the conclusions. Ref. [31] have studied the constraints on neutralino WIMPs with different annihilation channels. If we use their results (which have not been done for the new velocity measurements), the conclusion for the case of SD proton-only couplings is that virtually all neutralinos are incompatible with the combination of SuperK plus all direct detection experiments; this statement assumes that there is no WIMP/antiWIMP asymmetry as well as the standard annihilation cross section required for thermal WIMPs. [Note that Super-K would rule out SD proton-only couplings, not neutron-only or SI couplings.]

The low mass region found in the SI and the SD proton-only cases persists with a combination of these two couplings. That is, there are portions of parameter space with both SI couplings and SD couplings to the proton that are compatible with all the direct detection experiments.

Neutron only $\left(a_{p}=0\right)$ : Figure 5 shows the best fit DAMA regions for spin-dependent couplings for neutron only $\left(a_{p}=0\right)$. Figure 6 shows that there is no region of WIMP masses which can be compatible with all experiments in this case. While this SD neutron-only case is incompatible, non-zero SD couplings to the neutron are not in general incompatible: cases with mixed couplings (with both $a_{p} \neq 0$ and $a_{n} \neq 0$ ) can still be found that are consistent with all the experimental results. 

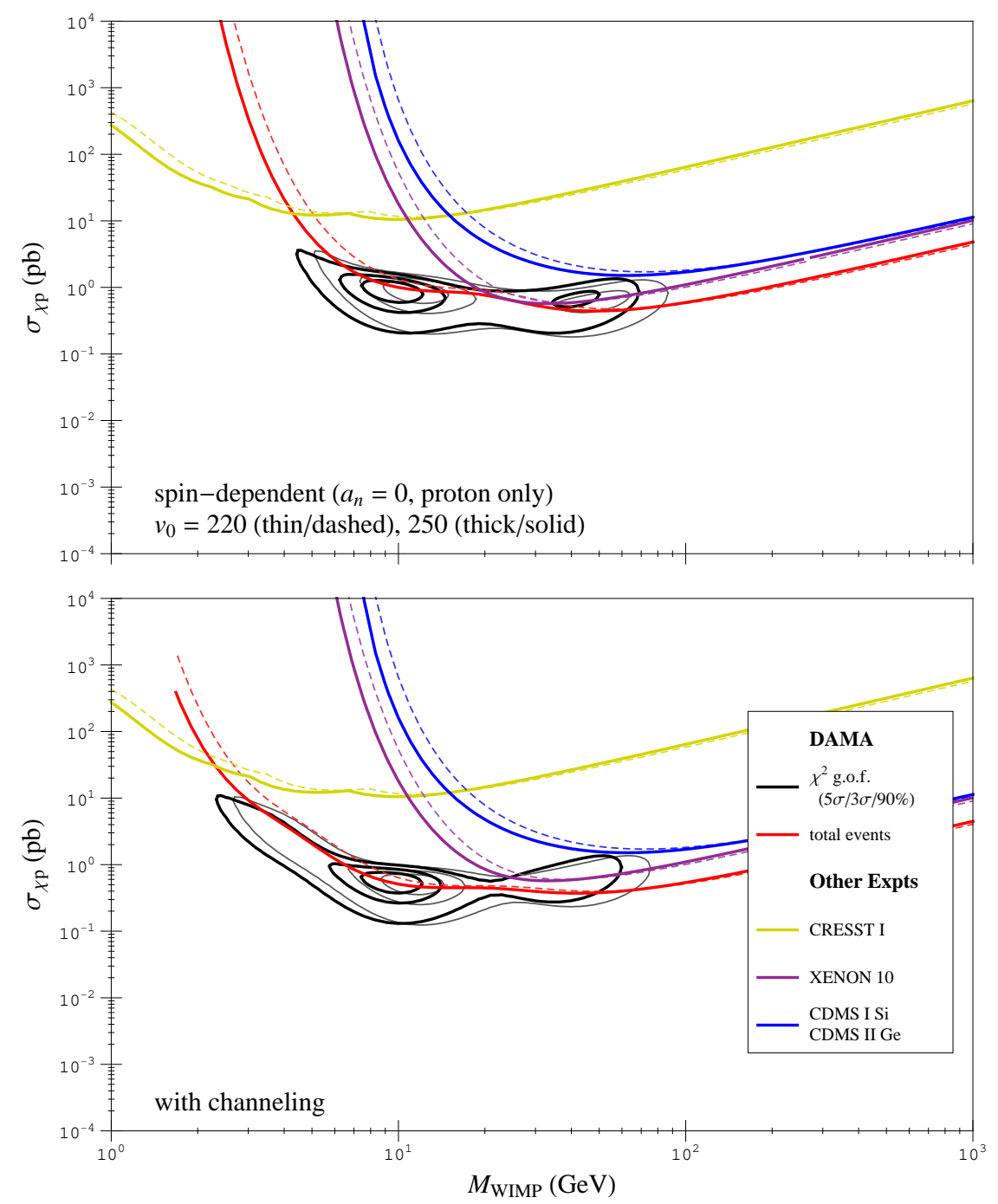

FIG. 4: Same as Figure 2 but for SD proton-only scattering $\left(a_{n}=0\right)$. Without Super-K included compatiblity is viable for 5-15 GeV WIMP masses for direct detection experiments (but has been argued by others to be further constrained by Super-Kamiokande due to annihilation in the Sun).

\section{CONCLUSION}

Our general result is that the new determination of the local standard of rest moves the best fit WIMP masses to the DAMA data to lower values. We had hoped that the DAMA data, which is very dependent on the WIMP velocity behavior, would change enough to allow new regions of parameter space compatible with all experiments. However, the bounds from the other experiments shifted by about the same amount, so that very little changed due to the analysis in this paper.

\section{Acknowledgments}

We acknowledge the Michigan Center for Theoretical Physics at the University of Michigan, where this project came into existence while we were all attending the workshop on 

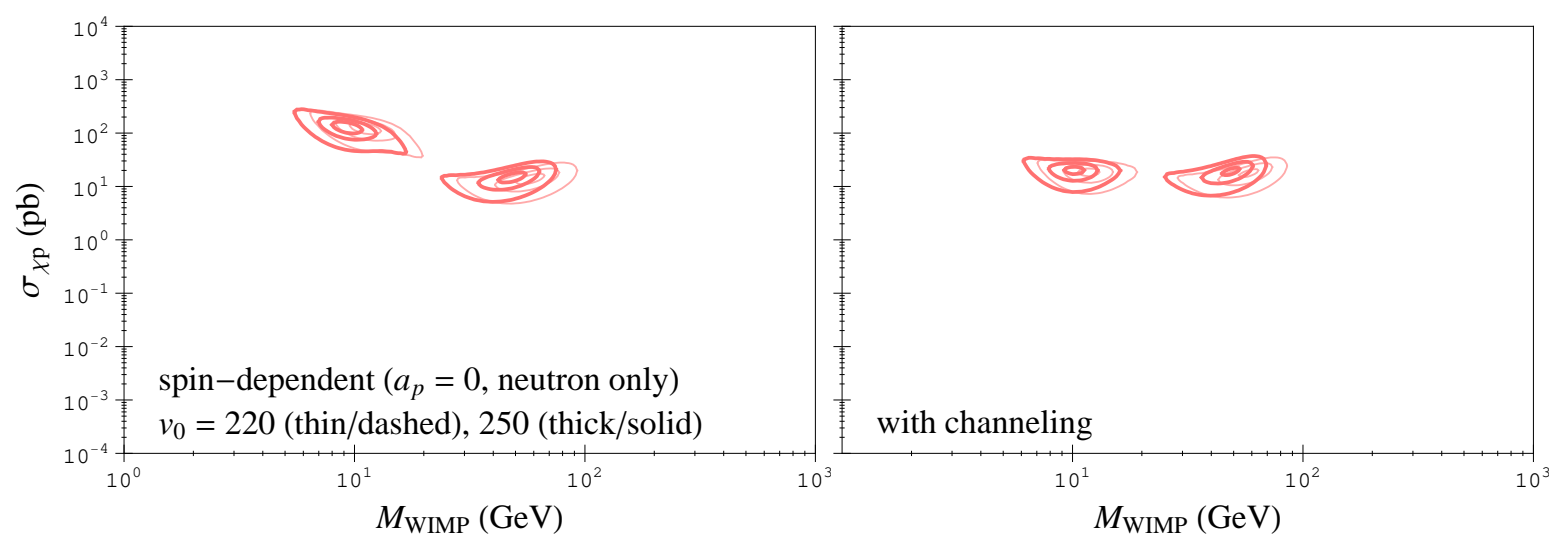

FIG. 5: Same as Figure 1 but for SD neutron-only scattering $\left(a_{p}=0\right)$.
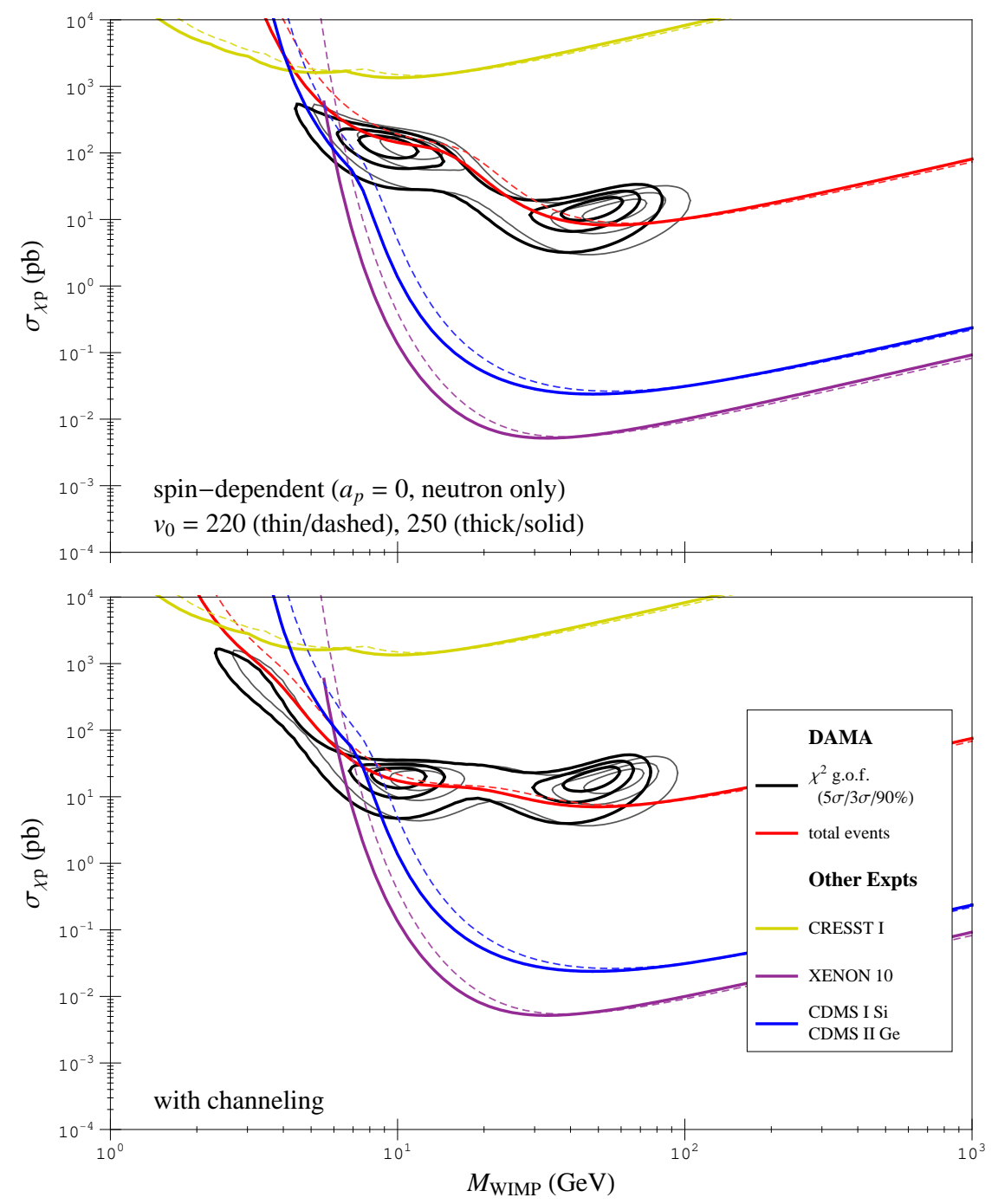

FIG. 6: Same as Figure 2 but for SD neutron-only scattering $\left(a_{p}=0\right)$. No regions of parameter space remain. 
LHC and Dark Matter. K.F. acknowledges the support of the DOE via the University of Michigan. P.G. was partially supported by NSF grant PHY-0756962 at the University of Utah. C.S. acknowledges the support of the William I. Fine Theoretical Physics Institute at the University of Minnesota. D.S. acknowledges a GAANN fellowship.

[1] M.Reid, http://online.itp.ucsb.edu/online/milkyway08/reid/

[2] R. Bernabei et al., Riv. Nuovo Cim. 26N1, 1 (2003) arXiv:astro-ph/0307403.

[3] R. Bernabei et al. [DAMA Collaboration], Eur. Phys. J. C 56, 333 (2008) [arXiv:0804.2741 [astro-ph]].

[4] A. K. Drukier, K. Freese and D. N. Spergel, Phys. Rev. D 33, 3495 (1986).

[5] K. Freese, J. A. Frieman and A. Gould, Phys. Rev. D 37, 3388 (1988).

[6] G. Gelmini and P. Gondolo, arXiv:hep-ph/0405278.

[7] P. Gondolo and G. Gelmini, Phys. Rev. D 71, 123520 (2005) arXiv:hep-ph/0504010.

[8] D. S. Akerib et al. [CDMS Collaboration], Phys. Rev. D 68, 082002 (2003) arXiv:hep-ex/0306001.

[9] Z. Ahmed et al. [CDMS Collaboration], Phys. Rev. Lett. 102, 011301 (2009) arXiv:0802.3530 [astro-ph]].

[10] C. E. Aalseth et al. [CoGeNT Collaboration], Phys. Rev. Lett. 101, 251301 (2008) [Erratumibid. 102, 109903 (2009)] arXiv:0807.0879 [astro-ph]].

[11] E. Behnke et al. [COUPP Collaboration], Science 319, 933 (2008) [arXiv:0804.2886][astro-ph]].

[12] G. Angloher et al., Astropart. Phys. 18, 43 (2002).

[13] L. Stodolsky and F. Probst, talk at "The Dark Side of the Universe," Ann Arbor, May 2004.

[14] S. T. Lin et al. [TEXONO Collaboration], Phys. Rev. D 79, 061101 (2009) arXiv:0712.1645 [hep-ex]].

[15] J. Angle et al. [XENON Collaboration], Phys. Rev. Lett. 100, 021303 (2008) arXiv:0706.0039 [astro-ph]].

[16] J. Angle et al., Phys. Rev. Lett. 101, 091301 (2008) [arXiv:0805.2939 [astro-ph]].

[17] M. Brhlik and L. Roszkowski, Phys. Lett. B 464, 303 (1999) arXiv:hep-ph/9903468; C. J. Copi and L. M. Krauss, Phys. Rev. D 67, 103507 (2003) [arXiv:astro-ph/0208010]; R. Foot, Phys. Rev. D 69, 036001 (2004) arXiv:hep-ph/0308254]; R. Foot, Mod. Phys. Lett. A 19, 1841 (2004) arXiv:astro-ph/0405362; A. M. Green, Phys. Rev. D 63, 043005 (2001) arXiv:astro-ph/0008318]; A. M. Green, Phys. Rev. D 66, 083003 (2002) arXiv:astro-ph/0207366]; A. M. Green, Phys. Rev. D 68, 023004 (2003) [Erratum-ibid. D 69, 109902 (2004)] arXiv:astro-ph/0304446]; G. Prezeau, A. Kurylov, M. Kamionkowski and P. Vogel, Phys. Rev. Lett. 91, 231301 (2003) arXiv:astro-ph/0309115; P. Ullio, M. Kamionkowski and P. Vogel, JHEP 0107, 044 (2001) arXiv:hep-ph/0010036; A. Kurylov and M. Kamionkowski, Phys. Rev. D 69, 063503 (2004) arXiv:hep-ph/0307185]; F. Giuliani and T. A. Girard, Phys. Rev. D 71, 123503 (2005) arXiv:hep-ph/0502232.

[18] J. Silk, K. A. Olive and M. Srednicki, Phys. Rev. Lett. 55, 257 (1985); K. Freese and M. Kamionkowski, Phys. Rev. D 55, 1771 (1997) arXiv:hep-ph/9609370].

[19] K. Freese, Phys. Lett. B 167, 295 (1986); L. M. Krauss, M. Srednicki and F. Wilczek, Phys. Rev. D 33, 2079 (1986).

[20] S. Desai et al. [Super-Kamiokande Collaboration], Phys. Rev. D 70, 083523 (2004) [Erratumibid. D 70, 109901 (2004)] [arXiv:hep-ex/0404025]. 
[21] C. Savage, P. Gondolo and K. Freese, Phys. Rev. D 70, 123513 (2004) arXiv:astro-ph/0408346].

[22] R. Bernabei et al., Int. J. Mod. Phys. D 13, 2127 (2004) arXiv:astro-ph/0501412.

[23] A. M. Green, Phys. Rev. D 68, 023004 (2003) [Erratum-ibid. D 69, 109902 (2004)] [arXiv:astro-ph/0304446].

[24] A. Bottino, F. Donato, N. Fornengo and S. Scopel, Phys. Rev. D 78, 083520 (2008) arXiv:0806.4099 [hep-ph]].

[25] D. Tucker-Smith and N. Weiner, Phys. Rev. D 64, 043502 (2001) arXiv:hep-ph/0101138.

[26] C. Savage, G. Gelmini, P. Gondolo and K. Freese, JCAP 0904, 010 (2009) arXiv:0808.3607 [astro-ph]].

[27] F. Petriello and K. M. Zurek, JHEP 0809, 047 (2008) arXiv:0806.3989 [hep-ph]].

[28] S. Chang, G. D. Kribs, D. Tucker-Smith and N. Weiner, Phys. Rev. D 79, 043513 (2009) arXiv:0807.2250 [hep-ph]].

[29] S. Chang, A. Pierce and N. Weiner, arXiv:0808.0196 [hep-ph].

[30] M. Fairbairn and T. Schwetz, JCAP 0901, 037 (2009) arXiv:0808.0704 [hep-ph]].

[31] D. Hooper, F. Petriello, K. M. Zurek and M. Kamionkowski, Phys. Rev. D 79, 015010 (2009) arXiv:0808.2464 [hep-ph]].

[32] S. Andreas, T. Hambye and M. H. G. Tytgat, JCAP 0810, 034 (2008) arXiv:0808.0255 [hep-ph]].

[33] R. Foot, Phys. Rev. D 78, 043529 (2008) [arXiv:0804.4518 [hep-ph]].

[34] M. Y. Khlopov and C. Kouvaris, Phys. Rev. D 78, 065040 (2008) arXiv:0806.1191 [astro-ph]]; M. Y. Khlopov, arXiv:0806.3581 [astro-ph]; C. Kouvaris, Phys. Rev. D 78, 075024 (2008) arXiv:0807.3124 [hep-ph]].

[35] J. L. Feng, J. Kumar and L. E. Strigari, Phys. Lett. B 670, 37 (2008) arXiv:0806.3746 [hep-ph]].

[36] K. L. J. Rygl, A. Brunthaler, K. M. Menten, M. J. Reid and H. J. van Langevelde, arXiv:0812.0905 [astro-ph].

[37] M. J. Reid, K. M. Menten, A. Brunthaler, X. W. Zheng, L. Moscadelli and Y. Xu, Astrophys. J. 693, 397 (2009) arXiv:0811.0595 [astro-ph]]; L. Moscadelli, M. J. Reid, K. M. Menten, A. Brunthaler, X. W. Zheng and Y. Xu, Astrophys. J. 693, 406 (2009) arXiv:0811.0679 [astro-ph]]; Y. Xu, M. J. Reid, K. M. Menten, A. Brunthaler, X. W. Zheng and L. Moscadelli, Astrophys. J. 693, 413 (2009) arXiv:0811.0701 [astro-ph]]; B. Zhang, X. W. Zheng, M. J. Reid, K. M. Menten, Y. Xu, L. Moscadelli and A. Brunthaler, Astrophys. J. 693, 419 (2009) arXiv:0811.0704 [astro-ph]]; A. Brunthaler, M. J. Reid, K. M. Menten, X. W. Zheng, L. Moscadelli and Y. Xu, Astrophys. J. 693, 424 (2009) [arXiv:0811.0713 [astro-ph]].

[38] M. Honma et al., arXiv:0709.0820 [astro-ph].

[39] K. Hachisuka et al., Astrophys. J. 645, 337 (2006) arXiv:astro-ph/0512226.

[40] M. W. Goodman and E. Witten, Phys. Rev. D 31, 3059 (1985).

[41] G. Jungman, M. Kamionkowski and K. Griest, Phys. Rept. 267, 195 (1996) arXiv:hep-ph/9506380.

[42] P. F. Smith and J. D. Lewin, Phys. Rept. 187, 203 (1990); J. D. Lewin and P. F. Smith, Astropart. Phys. 6, 87 (1996).

[43] G. Duda, A. Kemper and P. Gondolo, JCAP 0704, 012 (2007) arXiv:hep-ph/0608035.

[44] J. Engel, Phys. Lett. B 264, 114 (1991).

[45] P. Gondolo, in XXXI Rencontres de Moriond: Dark Matter in Cosmology, Quantum Measurements, Experimental Gravitation, Les Arcs, France, 1996 arXiv:hep-ph/9605290. 
[46] D. R. Tovey, R. J. Gaitskell, P. Gondolo, Y. Ramachers and L. Roszkowski, Phys. Lett. B 488, 17 (2000) arXiv:hep-ph/0005041.

[47] P. Gondolo, J. Edsjo, P. Ullio, L. Bergstrom, M. Schelke and E. A. Baltz, JCAP 0407, 008 (2004) arXiv:astro-ph/0406204.

[48] J. Engel, M. T. Ressell, I. S. Towner and W. E. Ormand, Phys. Rev. C 52, 2216 (1995) arXiv:hep-ph/9504322.

[49] M. T. Ressell and D. J. Dean, Phys. Rev. C 56, 535 (1997) arXiv:hep-ph/9702290].

[50] M. T. Ressell, M. B. Aufderheide, S. D. Bloom, K. Griest, G. J. Mathews and D. A. Resler, Phys. Rev. D 48, 5519 (1993).

[51] V. A. Bednyakov and F. Simkovic, Phys. Part. Nucl. 37, S106 (2006) arXiv:hep-ph/0608097.

[52] K. Freese, P. Gondolo and H. J. Newberg, Phys. Rev. D 71, 043516 (2005) arXiv:astro-ph/0309279].

[53] G. Gelmini and P. Gondolo, Phys. Rev. D 64, 023504 (2001) arXiv:hep-ph/0012315.

[54] C. Savage, K. Freese and P. Gondolo, Phys. Rev. D 74, 043531 (2006) arXiv:astro-ph/0607121].

[55] R. Bernabei et al., Eur. Phys. J. C 53, 205 (2008) [arXiv:0710.0288 [astro-ph]].

[56] J. Ahrens et al. [AMANDA Collaboration], Phys. Rev. D 66, 032006 (2002) arXiv:astro-ph/0202370.

[57] M. Ackermann et al. [AMANDA Collaboration], Astropart. Phys. 24, 459 (2006) arXiv:astro-ph/0508518].

[58] J. Ahrens et al. [The IceCube Collaboration], Nucl. Phys. Proc. Suppl. 118, 388 (2003) arXiv:astro-ph/0209556.

[59] F. Blanc et al. [ANTARES Collaboration], Presented by L. Thompson on behalf of the ANTARES Collaboration, (ICRC 2003), Tsukuba, Japan, 31 Jul - 7 Aug 2003.

[60] J. Silk and M. Srednicki, Phys. Rev. Lett. 53, 624 (1984); J. R. Ellis, R. A. Flores, K. Freese, S. Ritz, D. Seckel and J. Silk, Phys. Lett. B 214, 403 (1988); F. W. Stecker, S. Rudaz and T. F. Walsh, Phys. Rev. Lett. 55, 2622 (1985).

[61] P. Gondolo and J. Silk, Phys. Rev. Lett. 83, 1719 (1999) arXiv:astro-ph/9906391.

[62] C. Amsler et al. [Particle Data Group], Phys. Lett. B 667 (2008) 1.

[63] F. James, Statistical methods in experimental physics, Hackensack, NJ: World Scientific, (2006). 